\section{P03.30 TUMUR MUTATIONS DRIVE DYSFUNCTIONAL T CELL DIFFERENTIATION IN LUNG CANCER}

${ }^{1} E$ Ghorani* ${ }^{*}$ ' I Reading, ${ }^{1} \mathrm{~J}$ Henry, ${ }^{1} \mathrm{M}$ Robert de Massy, ${ }^{2} \mathrm{R}$ Rosenthal, ${ }^{3} \mathrm{~V}$ Turati, ${ }^{1} \mathrm{~A}$ Furness, ${ }^{1} \mathrm{~A}$ Ben Aissa, ${ }^{4} \mathrm{~S}$ Kumar Saini, ${ }^{4} \mathrm{~S}$ Ramskov, ${ }^{1} \mathrm{~A}$ Georgiou, ${ }^{1} \mathrm{M}$ Vila De Mucha, ${ }^{1}$ I Uddin, ${ }^{5} \mathrm{~T}$ Ronel, ${ }^{6} \mathrm{R}$ Salgado, ${ }^{1} \mathrm{~T}$ Lund, ${ }^{7} \mathrm{~J}$ Herrero, ${ }^{3} \mathrm{~T}$ Enver, ${ }^{8} \mathrm{~S}$ Hadrup, ${ }^{9} \mathrm{~A}$ Hackshaw, ${ }^{1} \mathrm{~K}$ Peggs, ${ }^{2} \mathrm{~N}$ McGranahan, ${ }^{5} \mathrm{~B}$ Chain, ${ }^{2} \mathrm{C}$ Swanton, ${ }^{1} \mathrm{~S}$ Quezada. ${ }^{1}$ Cancer Immunology Unit, UCL Cancer Institute, London, UK; ${ }^{2}$ Cancer Research UK Lung Cancer Centre of Excellence, UCL Cancer Institute, London, UK; ${ }^{3}$ Department of Cancer Biology, UCL Cancer Institute, London, UK; ${ }^{4}$ Department of Health Technology, Technical University of Denmark, Lyngby, Denmark; ${ }^{5}$ Division of Infection and Immunity, UCL, London, UK; ${ }^{6}$ Department of Pathology, GZA-ZNA, Antwerp, Belgium; ' Bill Lyons Informatics Centre, UCL Cancer Institute, London, UK; ${ }^{8}$ Department of Health Technology, Technical University of Denmark, London, UK; ${ }^{9}$ Cancer Research UK andUCL Cancer Trials Centre, London, UK

\subsection{6/jitc-2020-ITOC7.68}

Background Effective anti-tumour immunity requires cancer antigen expression, but persistent antigen exposure in chronic viral infections and autoimmunity has a detrimental effect on immune function. This is associated with a decline of early differentiated $\mathrm{T}$ cell populations in favour of later differentiated, dysfunctional subsets, resulting in an unfavourable skewing of the immune landscape. It is unknown whether this occurs locally within the antigen rich tumour microenvironment, driving immune failure.

Materials and Methods We combined tumour infiltrating lymphocyte (TIL) high dimensional flow cytometry, bulk exome and RNA sequencing data from multiregional samples obtained from surgically resected tumours of treatment naive patients with non-small cell lung cancer (NSCLC) amongst the first 100 recruited to the prospective, UK-wide lung TRACERx study. Clonal relationship between $\mathrm{T}$ cell populations was determined by $\mathrm{T}$ cell receptor (TCR) sequencing. We additionally analysed publically available single $\mathrm{T}$ cell RNA sequencing data and bulk RNA sequencing data within TCGA.

Results $\mathrm{T}$ cell differentiation skewing $\left(\mathrm{T}^{\mathrm{DS}}\right)$ occurred amongst TILs in association with tumour mutational burden (TMB). Surprisingly, this was most evident within the CD4 compartment that had a greater abundance of central memory cells expressing the key transcription factor TCF7. Amongst CD4 cells, loss of a PD1 ${ }^{-} \mathrm{CCR} 7^{+} \mathrm{T}$ central memory population was accompanied by gain in abundance of PD1+ populations with exhausted (CD57 ${ }^{-} \mathrm{ICOS}^{\text {hi }} \mathrm{CTLA}^{\mathrm{hi}}{ }^{\mathrm{h}}$ ) and terminally differentiated effector $\left(\mathrm{CD} 57^{+}\right.$Eomes $\left.^{+}\right)$features. TCR sequencing revealed early and dysfunctional differentiated populations to be clonally related and CDR3 clustering analysis showed greater similarity of sequences shared vs. non-shared between subsets, consistent with an antigen driven differentiation process. Similar patterns were observed within the CD8 compartment. Identification of these subsets within single $\mathrm{T}$ cell RNA sequencing data revealed shared and distinct functional regulators, suggesting the enhanced effector capability of early compared to dysfunctionally differentiated populations. A validated transcriptional signature of $\mathrm{T}^{\mathrm{DS}}$ generated using TRACERx samples with paired flow cytometry and RNA sequencing data reflected loss of gene expression downstream of TCF7, and predicted worse survival within TRACERx and multiple TCGA cohorts including lung adenocarcinoma (LUAD).

Conclusions Our finding support a model of neoantigen driven $\mathrm{T}$ cell differentiation within the tumour microenvironment that drives the depletion of progenitor-like cells and gain in abundance of dysfunctional subsets, resulting in a loss of immune fitness. Our analysis of transcriptomic data elucidates potential regulatory mechanisms and therapeutic targets within the subsets identified.

Disclosure Information E. Ghorani: None. J. Reading: None. J. Henry: None. M. Robert de Massy: None. R. Rosenthal: E. Ownership Interest (stock, stock options, patent or other intellectual property); Modest; Achilles Therapeutics. F. Consultant/Advisory Board; Modest; Achilles Therapeutics. V. Turati: None. A. Furness: None. A. Ben Aissa: None. S. Kumar Saini: None. S. Ramskov: None. A. Georgiou: None. M. Vila De Mucha: None. I. Uddin: None. T. Ronel: None. R. Salgado: None. T. Lund: None. J. Herrero: None. T. Enver: None. S. Hadrup: None. A. Hackshaw: None. K. Peggs: E. Ownership Interest (stock, stock options, patent or other intellectual property); Modest; Achilles Therapeutics. N. McGranahan,: E. Ownership Interest (stock, stock options, patent or other intellectual property); Modest; Achilles Therapeutics. F. Consultant/Advisory Board; Modest; Achilles Therapeutics. B. Chain: None. C. Swanton: B. Research Grant (principal investigator, collaborator or consultant and pending grants as well as grants already received); Modest; Pfizer, AstraZeneca, BMS, Roche-Ventana and Boehringer Ingelheim. E. Ownership Interest (stock, stock options, patent or other intellectual property); Modest; ApoGen Biotechnologies, Epic Bioscience and GRAIL, and has stock options in and is co-founder of Achilles Therapeutics. F. Consultant/Advisory Board; Modest; Pfizer, Novartis, GlaxoSmithKline, MSD, BMS, Celgene, AstraZeneca, Illumina, Genentech, Roche-Ventana, GRAIL, Medicxi and the Sarah Cannon Research Institute. S. Quezada: E. Ownership Interest (stock, stock options, patent or other intellectual property); Modest; Achilles Therapeutics.

\section{P03.31 SKIN DENDRITIC CELLS IN MELANOMA ARE KEY FOR SUCCESSFUL CHECKPOINT BLOCKADE THERAPY}

${ }^{1} \mathrm{~N}$ Prokopi ${ }^{*},{ }^{2} \mathrm{CH}$ Tripp, ${ }^{3} \mathrm{~B}$ Tummers, ${ }^{3} \mathrm{JC}$ Crawford, ${ }^{2} \mathrm{M}$ Efremova, ${ }^{2} \mathrm{~K}$ Hutter, ${ }^{2} \mathrm{~L}$ Bellmann, ${ }^{2} \mathrm{G}$ Cappellano, ${ }^{4} \mathrm{~L}$ Boon, ${ }^{2} \mathrm{D}$ Ortner, ${ }^{2} \mathrm{Z}$ Trajanoski, ${ }^{5} \mathrm{~S}$ Chen, ${ }^{6} \mathrm{~T}$ de Gruijl, ${ }^{3} \mathrm{DR}$ Green, ${ }^{2} \mathrm{P}$ Stoitzner. 'Amsterdam UMC, VUMC, Amsterdam, Netherlands; ${ }^{2}$ Medical University of Innsbruck, Innsbruck, Austria; ${ }^{3}$ St. Jude Children's Research Hospital, Memphis, TN, USA; ${ }^{4}$ Bioceros BV, Utrecht, Netherlands; ${ }^{5}$ Rutgers University, Piscataway, NJ, USA; ${ }^{6}$ Vrije Universiteit, Amsterdam, Netherlands

\subsection{6/jitc-2020-ITOC7.69}

Background Immunotherapy of cancer by checkpoint blockade has significantly improved the survival of melanoma patients. However, in patients with tumors that are poorly infiltrated by effector $\mathrm{T}$ cells the clinical results are not encouraging. Therefore, combination approaches that enhance pre-existing anti-tumor immunity and reset the patients' immunological status are urgently needed. In this study we used the $\operatorname{tg}(\mathrm{Grm} 1) \mathrm{EPv}$ melanoma mouse model that reflects a non-immunogenic tumor microenvironment. In this mouse model, spontaneous melanoma development is driven by the ectopic expression of the metabotropic glutamate receptor-1 in melanocytes, which confers to them a hyperproliferative and anti-apoptotic phenotype. The same alteration has been shown to be present in $40 \%$ of melanoma patient samples. The aim of our study was to investigate whether enhancing dendritic cell (DC) numbers and function in the $\operatorname{tg}(\mathrm{Grm} 1) \mathrm{EPv}$ mouse model could restore responsiveness to checkpoint blockade.

Material and Methods We used multicolor flow cytometry, gene expression analysis by RNA-seq and microarray to 